\title{
Investigating the nature of INTEGRAL Gamma-ray Bursts and sub-threshold triggers with Swift follow-up
}

\author{
A. B. Higgins, ${ }^{1}$ R. L. C. Starling, ${ }^{1}$ D. Götz, ${ }^{2}$ S. Mereghetti, ${ }^{3}$ K. Wiersema, ${ }^{1}$ \\ T. Maccarone, ${ }^{4}$ J. P. Osborne, ${ }^{1}$ N. R. Tanvir, ${ }^{1}$ P. T. O'Brien, ${ }^{1}$ A. J. Bird,${ }^{5}$ \\ A. Rowlinson ${ }^{6,7}$ and N. Gehrels ${ }^{8}$ \\ ${ }^{1}$ Department of Physics and Astronomy, University of Leicester, University Road, Leicester LE1 7RH, UK. \\ ${ }^{2}$ AIM-CEA/DRF/Irfu/Service d'Astrophysique, Orme des Merisiers, 91191 Gif-sur-Yvette, France. \\ ${ }^{3}$ INAF, IASF-Milano, via E.Bassini 15, 20133 Milano, Italy. \\ ${ }^{4}$ Department of Physics and Astronomy, Texas Tech University, Box 41051, Lubbock, TX 79409, USA. \\ ${ }^{5}$ School of Physics and Astronomy, University of Southampton, Southampton SO17 1BJ, UK. \\ ${ }^{6}$ Netherlands Institute for Radio Astronomy (ASTRON), PO Box 2, 7990 AA Dwingeloo, The Netherlands. \\ ${ }^{7}$ Anton Pannekoek Institute, University of Amsterdam, Postbus 94249, 1090 GE, Amsterdam, The Netherlands. \\ ${ }^{8}$ NASA Goddard Space Flight Center, Greenbelt, MD 20771, USA.
}

Accepted . Received ; in original form .

\begin{abstract}
We explore the potential of INTEGRAL to improve our understanding of the low fluence regime for explosive transients, such as GRBs. We probe the nature of the so-called "WEAK" INTEGRAL triggers, when the gamma-ray instruments record intensity spikes that are below the usual STRONG significance thresholds. In a targeted $S$ wift follow-up campaign, we observed 15 WEAK triggers. We find six of these can be classified as GRBs. This includes GRB 150305A, a GRB discovered from our campaign alone. We also identified a source coincident with one trigger, IGRW 151019, as a candidate AGN. We show that real events such as GRBs exist within the IBAS WEAK trigger population. A comparison of the fluence distributions of the full INTEGRAL IBAS and Swift BAT GRB samples showed that the two are similar. We also find correlations between the prompt gamma-ray and X-ray properties of the two samples, supporting previous investigations. We find that both satellites reach similar, low fluence levels regularly, although Swift is more sensitive to short, low fluence GRBs.
\end{abstract}

Key words: gamma-ray burst;general

\section{INTRODUCTION}

GRBs are among the most luminous events in the universe, releasing energies $>10^{51}$ erg typically in time periods of seconds (Gehrels \& Mészáros 2012). During these events a huge amount of gravitational energy is released from a central engine which leads to the formation of jets where particles are accelerated to ultra-relativistic speeds (Woosley \& Heger 2006). Internal shocks within the jet produce the high energy prompt gamma-ray emission we first observe (Gehrels \& Mészáros 2012; Piran 2003). The jet then shocks with the surrounding medium producing broad-band afterglow emission (Mészáros \& Rees 1997; Wijers et al. 1997). Classically, GRBs are split into two sub-groups based on their $\mathrm{T}_{90}$ - the duration over which 90 per cent of the gamma-ray flux is received (Kouveliotou et al. 1993). The two groups are short GRBs where $\mathrm{T}_{90}<2 \mathrm{~s}$ and long GRBs where $\mathrm{T}_{90}>2 \mathrm{~s}$, linked with two different progenitor models.

GRBs span a large range of isotropic equivalent luminosities: $10^{45} \leq \mathrm{L}_{\mathrm{ISO}} \leq 10^{54} \mathrm{erg} \mathrm{s}^{-1}$. Investigations into their luminosity function and formation rates coupled with observations of several local GRBs (Sazonov et al. 2004; Soderberg et al. 2004) have suggested that there should be a large number of low-luminosity GRBs (Daigne \& Mochkovitch 2007; Liang et al. 2007; Pescalli et al. 2016). There are further suggestions that these could exist as a separate local population (Norris 2002; Norris et al. 2005; Chapman et al. 2007; Liang et al. 2007). We use two currently active GRB detecting missions, Swift (Gehrels et al. 2004) and The INTErnational Gamma-Ray Astrophysics Laboratory (Winkler et al. 2003; INTEGRAL), to look at potentially faint GRBs.

INTEGRAL carries two gamma-ray instruments, IBIS (Ubertini et al. 2003) and SPI (Vedrenne et al. 2003). Alerts for GRBs and other transient sources are communicated with low latency by the INTEGRAL Burst Alert System, IBAS ${ }^{1}$ (Mereghetti et al. 2003) discussed in more detail in section 2. Since the launch in 2002

\footnotetext{
1 http://ibas.iasf-milano.inaf.it/
} 
INTEGRAL has detected over 900 soft gamma-ray sources ${ }^{2}$ (Bird et al. 2016) and has localised 114 GRBs (numbers correct as of 2016 July 1). INTEGRAL has made some important discoveries regarding GRBs, reviewed in Götz (2013) including investigations utilising IBIS and SPIs capability to perform spectral analysis on the INTEGRAL sample of GRBs (Vianello et al. 2009; Bošnjak et al. 2014). Furthermore, Foley et al. (2008) suggested that INTE$G R A L$ may be capable of detecting the local, low-luminosity GRB populations.

In the fully coded field of view (FOV), i.e. the central $9^{\circ} \times 9^{\circ}$, the INTEGRAL IBIS instrument is more sensitive than the Burst Alert Telescope (BAT) (Barthelmy 2004) on board Swift, despite its smaller effective area $\left(2600 \mathrm{~cm}^{2}\right.$ compared to $\left.5200 \mathrm{~cm}^{2}\right)$. This is due to the fact that, at the energies we are interested in $(15-200$ $\mathrm{keV}$ ), the background is dominated by the Cosmic X-ray diffuse emission, which is proportional to the FOV (a factor of about ten smaller for IBIS than for BAT). Therefore INTEGRAL should be able to reach lower peak flux limits, especially for GRBs with hard spectra where peak energies $>50 \mathrm{keV}$ (Bošnjak et al. 2014). However, since INTEGRAL spends a large fraction of its observing time observing at low Galactic latitudes, its sensitivity is reduced by the additional background caused by bright Galactic sources and hard $\mathrm{X}$-ray Galactic diffuse emission. It is only since the INTEGRAL sub-threshold trigger campaign began (see section 2) that lower sensitivities have been routinely accessible through WEAK alerts.

Swift has two additional instruments, the X-Ray Telescope (XRT) (Burrows et al. 2005) and the Ultraviolet/Optical Telescope (UVOT) (Roming et al. 2005), and has the ability to slew towards a BAT-detected burst or pre-selected target. Therefore it can complement INTEGRAL with rapid multi-wavelength, follow-up measurements. Using observations from both satellites we expect to uncover both the temporal behaviour and energetics of both the WEAK alerts and INTEGRAL GRB sample and characterise their properties.

We start by discussing the INTEGRAL Burst Alert System (IBAS) in more detail and describe our chosen WEAK triggers in section 2. Our Swift follow-up analysis is discussed in section 3. These are then analysed in conjunction with the total IBAS GRB sample in section 4 with some comparisons to the Swift GRB population. We conclude with our summary in section 5 .

\section{INTEGRAL IBAS ALERTS}

INTEGRAL was designed as a general purpose gamma-ray observatory, not specifically optimized for the study of GRBs. However, its good imaging capabilities over a field of view of $\approx 30 \times 30^{\circ}$ $\left(9 \times 9^{\circ}\right.$ fully coded and $19 \times 19^{\circ}$ half coded $)$ and the continuous telemetry downlink (due to its high elliptical orbit with a period of 3 days) made it possible to search and localize GRBs on the ground in near real time. This is done with the INTEGRAL Burst Alert System, IBAS (Mereghetti et al. 2003), software running at the $I N$ TEGRAL Science Data Centre, ISDC (Courvoisier et al. 2003) since the launch of INTEGRAL in October 2002.

No GRB triggering algorithm is implemented on board the satellite. The data reach the ISDC typically within 20 seconds after they have been collected and are immediately fed into the IBAS software which exploits several burst detection programs in parallel. When a burst (or any other new transient source) is detected inside

2 http://www.isdc.unige.ch/integral/ the field of view of the IBIS instrument, its coordinates are automatically distributed via the Internet by means of Alert Packets based on the User Datagram Protocol (UDP). Their coordinates derived by IBAS have a mean with $1 \sigma$ uncertainty of $2.1( \pm 0.5)$ arcmin.

IBAS also searches for GRBs detected in the Anti-Coincidence Shield (ACS) of the SPI instrument, which provides a good sensitivity over nearly the whole sky, but without localisation and spectral information (von Kienlin et al. 2003). The ACS lightcurves are used for GRB localizations by triangulation with other satellites of the IPN network (Cline et al. 1999). In this investigation we will not discuss SPI ACS results.

The search for GRBs in the IBIS data uses two different kinds of programs: rate monitor and image monitor programs. Rate monitors look for excesses in the light curve of the whole detection plane, while image monitors search for excesses in the deconvolved images. Both use data from ISGRI (Lebrun et al. 2003), the lower energy detector of IBIS, which provides photon by photon data in the energy range $15 \mathrm{keV}-1 \mathrm{MeV}$. Several instances of the rate and image monitors run in parallel using different settings for integration time scales and energy range. When one (or typically more) of the monitor programs triggers, an imaging analysis is performed on an optimally selected time interval in order to confirm the source presence and derive its significance.

Two significance threshold levels, labelled STRONG and WEAK, have been implemented in IBAS for what concerns the distribution of Alert Packets. The positions of new sources with significance above the STRONG threshold are immediately distributed with Alert Packets. These positions automatically derived by the IBAS software can be later refined by interactive analysis. Until 2011, Alert Packets for sources with significance above the WEAK threshold and below the STRONG were distributed in real time only to members of the IBAS Team, who, after interactive analysis could in some cases confirm the presence of a GRB and distribute its coordinates. However, in the majority of the cases it was not possible, based on the INTEGRAL data alone, to confirm the real astrophysical nature of these low significance events. Since 2011 January 26, all the Alert Packets corresponding to detections above the WEAK threshold have been automatically distributed in real time to the external users who wish to receive them.

Among the 114 confirmed GRBs detected by IBAS, 17 have been detected as sub-threshold WEAK alerts and 54 were observed with Swift, either through an independent autonomous BAT trigger and subsequent follow-up, or via $\mathrm{ToO}$ follow-up that was uploaded at a later time, and have available XRT data.

\subsection{Selection of WEAK alerts and follow-up}

There have been 402 INTEGRAL WEAK triggers, below $8 \sigma$ significance, before 2016 July 1 ; six of which were promoted to STRONG triggers and were later confirmed as GRBs. Out of the other 396 we analysed 15 WEAK triggers. They consisted of:

- 11 triggers that did not have prompt Swift slews and were target of opportunity observations from our campaign. We named them IGRWYYMMDD prior to source-type identification, broadly following the GRB naming convention, see table 1. These are termed as "our chosen ToOs".

- Two other WEAK INTEGRAL triggers with ToOs requested elsewhere and had XRT data, but were not related to our 11 chosen triggers, were analysed. These are termed as "candidate GRBs".

- Two WEAK triggers that also triggered BAT and had XRT data were also analysed. These are also termed as "candidate GRBs". 
Candidate triggers for our Swift ToO follow-up were selected with the requirement that at least one of the following criteria were met. Firstly, triggers were chosen to be close to the $8 \sigma$ STRONG threshold (our lowest was $6.7 \sigma$ ). This was to increase the chance of the trigger representing a real GRB. Trigger positions were also checked for high Galactic extinction and close proximity to nearby catalogued X-ray sources. Finally, triggers were generally only followed up if the trigger time coincided with the working hours of the on-call member of the Swift team. The criteria described above were not stringently adhered to for all triggers. We cannot claim that these triggers form a uniform or complete sample and biases towards high significance and lower Galactic column density are present. This was a pilot campaign aimed at determine whether real transient events exist among the WEAK trigger population and we stress that we do not make conclusions for the entire WEAK trigger population.

\section{SWIFT ANALYSIS}

The XRT and UVOT data from the 15 WEAK triggers with followup Swift observations discussed in section 2.1 were analysed to determine the nature of the WEAK trigger events. The data were made available by the UK Swift Science Data Centre (UKSSDC) (Evans et al. 2007, 2009).

Cleaned event files for our 11 ToOs were produced using the Swift XRT pipeline tool (v0.13.2). For the other four candidate GRBs we used the existing XRT products made available by the UKSSDC. For each $\mathrm{ToO}$ a search for any sources with a probability of being due to statistical fluctuations $<0.3$ per cent (equivalent to $3 \sigma$ ) within the INTEGRAL error region (90 per cent confidence) was conducted using the sky image file. Source counts were derived from 30 arcsec radius regions centred on any detected X-ray source coordinates. Upper limits on non-detections were also obtained using Bayesian analysis described in Kraft et al. (1991).

If a source was detected with the Swift XRT a further ToO observation was requested to identify whether the source was fading and thus could be confirmed as a GRB. If the source was detected again, and confirmed to be fading, a third observation was requested at a later date to check if the source had faded further. All positive detection coordinates were cross-referenced with the astrophysics catalogue database Vizier $^{3}$ to identify any existing sources that could account for the X-ray emission. We obtained the following results:

- For six of the 15 WEAK triggers, comprising of two of our chosen ToOs and the four candidate GRBs, we had a detection with the XRT. The Swift XRT properties of these events, along with the non-detections, can be found in table 2 .

- Subsequent observations found that five of these were fading X-ray sources, typical of a GRB afterglow (Costa et al. 1997; O'Brien et al. 2006) (see figure 5). The exception was IGRW 151019 (discussed in section 3.2).

- All 6 positive X-ray detections had no previously catalogued $\mathrm{X}$-ray sources within 2 arcmin at the time of the observations.

Two of the candidate GRBs, GRB 121212A and GRB 150831A, were relatively well observed by the XRT (> 10 data points) compared to the other WEAK trigger XRT sources as they also triggered BAT. These were further analysed

3 http://vizier.u-strasbg.fr/viz-bin/VizieR to obtain both a spectral fit and X-ray afterglow decay slope. The results can be seen in table 3 and figure 3 shows the spectrum for GRB 121212A. GRB 150831A has a $\mathrm{T}_{90} \approx 2 \mathrm{~s}$ - classifying it as a short GRB. Although ToO IGRW 110112 was an XRT non-detection at $6.2( \pm 0.6) \times 10^{4} \mathrm{~s}$ after the IBAS trigger, its initial gamma-ray trigger was seen simultaneously by Fermi GBM (Connaughton 2011) and so was classified as a GRB. IGRW 110608, one of the non-detections appeared to have an unusually high and irregular X-ray background compared to the other ToOs. This may have reduced our chances of getting a detection. The XRT non-detection upper limits can be seen in figure 2 .

In figure 4 we plot the time from the GRB to the start of the XRT observation $\left(T_{\text {START }}\right.$ ) against the weighted mean Galactic column density, $N_{\mathrm{H}}(\mathrm{Gal})$ for both detections and non-detections. As the X-ray emission decays over time a later observation may result in a non-detection of an X-ray source that had been present at an earlier time. Additionally, high Galactic column density may reduce the chance of achieving a detection. The values for $N_{\mathrm{H}}(\mathrm{Gal})$ were calculated using the method described in Willingale et al. (2013). Two sources observed less than $100 \mathrm{~s}$ after the initial trigger were both detected. Of the other 13 sources observed at later times after the triggers four were detected and nine were not. The column density and time since the trigger values for these detections and non-detections were similar and from our observations we saw that the column density (up to $\approx 10^{22} \mathrm{~cm}^{-2}$ ) and $T_{\text {START }}$ (up to $\approx 70000$ s) had no significant impact as to whether a WEAK trigger would be detected by the XRT. However, we only observed 15 sources and two sources observed within $100 \mathrm{~s}$ of the trigger were both detected so observing sources as promptly as possible would aid in detecting any potential afterglows.

For the six X-ray detections we analysed the UVOT data to determine if any UV/optical sources were present. The data comprised one or more UVOT filters each with a number of separate images. Multiple images were aligned and summed up to create one image per exposure for each filter. If there were multiple exposures over all observations of the ToO these were also additionally summed together to create one image with the total exposure over all observations. It must be noted that the number of filters used during each $\mathrm{ToO}$ exposure was dependent upon those already designated to be used by Swift on the date of the observation (the image binning could vary per exposure so only $1 \times 1$ binned images were used during the investigation - see Swift UVOT Online Manual).

To find the magnitude of a possible UVOT source, or upper limit on any non-detections, the Swift tool uvotsource was used with a significance of $3 \sigma$ to distinguish between a possible source and non-detection upper limit (Breeveld et al. 2010). From our UVOT analysis we found:

- An optical source was marginally detected $(<5 \sigma)$ with the UVOT white filter coincident with the XRT position of GRB 121212A. A Vizier search of the source position revealed no reported optical source. The UVOT position of the GRB 121212A optical source was RA, Dec (J2000) 177.79341, 78.03780 deg with a $1 \sigma$ positional error of 0.48 arcsec.

- A marginal detection was also registered in the $\mathrm{v}, \mathrm{b}$ and $\mathrm{u}$ bands for GRB 091111. Further analysis revealed that the event occurred within 30 arcsec of the centre of a very bright, saturated source which may have affected the background region near GRB 091111 resulting in a false detection.

- A UV source detected with the m2 filter $(9.2 \sigma)$ was present very close to the 90 per cent XRT error circle of IGRW 151019 (see section 3.2). 


\begin{tabular}{cccccc}
\hline $\begin{array}{c}\text { ToO } \\
\text { Name }\end{array}$ & $\begin{array}{c}\text { INTEGRAL } \\
\text { Trigger No. }\end{array}$ & $\begin{array}{c}\text { IBAS } \\
\text { detection } \\
\text { Significance } \\
(\sigma)\end{array}$ & $\begin{array}{c}\text { RA } \\
(\text { Deg }) \\
(J 2000)\end{array}$ & $\begin{array}{c}\text { Dec } \\
(\text { Deg }) \\
(J 2000)\end{array}$ & $\begin{array}{c}\text { Localisation } \\
\text { Error } \\
\text { (arcmin) }\end{array}$ \\
\hline IGRW 160610 & $7488 / 0$ & 6.7 & 359.90 & 61.57 & 3.8 \\
\hline IGRW 151019 & $7277 / 0$ & 7.0 & 292.82 & 31.14 & 3.5 \\
\hline IGRW 150903 & $7231 / 0$ & 6.7 & 239.17 & -33.81 & 3.6 \\
\hline IGRW 150610 & $7005 / 0$ & 7.1 & 178.32 & 16.03 & 4.8 \\
\hline IGRW 150305 & $6905 / 0$ & 7.6 & 269.79 & -42.62 & 3.4 \\
\hline IGRW 140219 & $6467 / 0$ & 6.7 & 204.10 & -45.06 & 3.6 \\
\hline IGRW 130904 & $6931 / 0$ & 6.7 & 256.88 & -32.01 & 3.6 \\
\hline IGRW 110718 & $6323 / 0$ & 6.8 & 256.78 & 40.05 & 3.6 \\
\hline IGRW 110608 & $6297 / 0$ & 6.8 & 315.28 & 32.041 & 3.6 \\
\hline IGRW 110428 & $6169 / 0$ & 7.2 & 320.27 & -33.96 & 3.5 \\
\hline IGRW 110112 & $6127 / 0$ & 7.4 & 10.56 & 64.41 & 2.6 \\
\hline IGRW 150831 & $7228 / 0$ & 7.3 & 220.98 & -25.65 & 3.4 \\
\hline IGRW 121212 & $6720 / 0$ & 7.9 & 177.90 & 78.00 & 3.3 \\
\hline IGRW 100909 & $6060 / 0$ & 7.7 & 73.95 & 54.65 & 2.0 \\
\hline IGRW 091111 & - & 7.2 & 137.81 & -45.91 & 2.9 \\
\hline
\end{tabular}

Table 1. Table containing the properties of the 15 WEAK triggers. The four triggers at the bottom are the four candidate GRBs with previous XRT observations and were not part of our selected ToOs. The Trigger No., significance $(\sigma)$, RA, Dec and localisation error ( 90 per cent confidence) were all taken from IBAS.

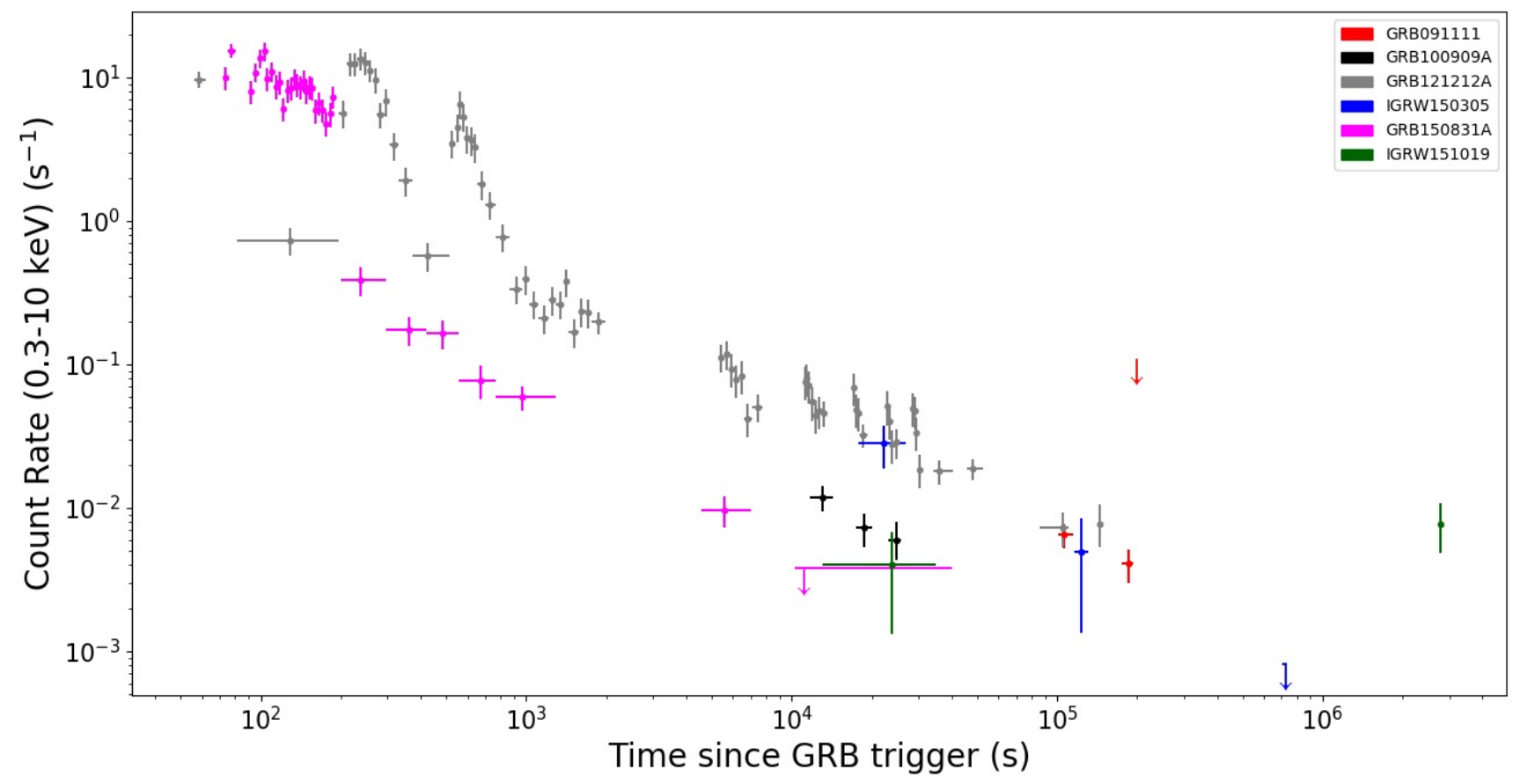

Figure 1. X-ray afterglows of the six WEAK INTEGRAL sources that were detected by Swift from our 15 WEAK triggers.

Table 4 contains the magnitudes and limits that were obtained for each source in each available filter. Some sources occurred in crowded fields affecting background subtraction. We also included Galactic reddening values for each source. The $A_{V}$ values were taken from the Infrared Science Archive (IRSA) ${ }^{4}$ using the method described in Schlafly \& Finkbeiner (2011).

4 http://irsa.ipac.caltech.edu/applications/DUST/

\subsection{GRB 150305A}

IGRW 150305 was confirmed to have a fading X-ray afterglow after requesting 3 ToOs over a time period of $\approx 8-9$ days, the first of which began $17 \mathrm{ks}$ after the WEAK trigger (see figure 5). A marginal detection was made in the white UVOT filter. A Vizier search of the GRB position revealed no optical or X-ray catalogue matches for the XRT and UVOT positions.

The light curve of GRB 150305A was poorly sampled due 


\begin{tabular}{cccccccc}
\hline $\begin{array}{c}\text { ToO } \\
\text { Name }\end{array}$ & $\begin{array}{c}\text { Swift } \\
\text { Obs ID }\end{array}$ & $\begin{array}{c}\text { XRT } \\
\text { Position } \\
\text { Error } \\
(\operatorname{arcsec})\end{array}$ & $\begin{array}{c}\text { RA } \\
\text { (Deg) } \\
(\mathrm{J} 2000)\end{array}$ & $\begin{array}{c}\text { Dec } \\
(\mathrm{Deg}) \\
(\mathrm{J} 2000)\end{array}$ & $\begin{array}{c}T_{\text {START }} \\
(\mathrm{s})\end{array}$ & $\begin{array}{c}T_{\text {STOP }} \\
(\mathrm{s})\end{array}$ & $\begin{array}{c}T_{\text {EXP }} \\
(\mathrm{s})\end{array}$ \\
\hline IGRW 151019 & 20558 & 2.5 & 292.7836 & 31.1319 & 9600 & 2810017 & 14938 \\
\hline GRB 150831A & 653838 & 1.6 & 221.0243 & -25.6351 & 82 & 38575 & 11828 \\
\hline IGRW 150305A & 33663 & 3.5 & 269.7606 & -42.6638 & 17838 & 735268 & 908 \\
\hline GRB 121212A & 541371 & 1.4 & 177.7923 & 78.0371 & 60 & 145420 & 22940 \\
\hline GRB 100909A & 20147 & 3.3 & 73.9488 & 54.6579 & 11693 & 25787 & 7720 \\
\hline GRB 091111 & 20120 & 7.7 & 137.8233 & -45.9253 & 100360 & 197466 & 10386 \\
\hline
\end{tabular}

Table 2. Swift XRT ToO observations and candidate counterpart source detections. XRT position (90 per cent confidence), RA and Dec were taken from UKSSDC. $T_{\mathrm{START}}$ refers to the time elapsed between the GRB/trigger occurring and the time when the Swift observation began and $T_{\mathrm{STOP}}$ refers to the time elapsed between the GRB occurring and the time when the final Swift observation finished. $T_{\mathrm{EXP}}$ is the total XRT exposure time.

\begin{tabular}{lccccc}
\hline \multicolumn{1}{c}{ ToO Name } & $\begin{array}{c}N_{\mathrm{H}}(\mathrm{Gal})\left(10^{20}\right. \\
\left.\mathrm{cm}^{-2}\right)\end{array}$ & $\begin{array}{c}N_{\mathrm{H}}(\mathrm{Int})\left(10^{20}\right. \\
\left.\mathrm{cm}^{-2}\right)\end{array}$ & $\Gamma$ & $\begin{array}{c}\text { C-Stat } \\
(\mathrm{dof})\end{array}$ & $\alpha$ \\
\hline GRB 121212A & 4.48 & $21_{-0.4}^{+0.5}$ & $2.24_{-0.13}^{+0.14}$ & $341(369)$ & $-0.71_{-0.03}^{+0.03}$ \\
\hline GRB 150831A (WT) & 11.4 & $0_{-0}^{+80.0}$ & $1.15_{-0.1}^{+0.18}$ & $322(404)$ & $-2.67_{-0.22}^{+0.22}$ \\
\hline GRB 150831A (PC) & 11.4 & $0_{-0}^{+18.0}$ & $1.53_{-0.29}^{+0.28}$ & $99(93)$ & $-2.67_{-0.22}^{+0.22}$ \\
\hline
\end{tabular}

Table 3. Table containing the X-ray spectral and afterglow light curve properties of WEAK GRBs with $>10$ binned data points. $N_{\mathrm{H}}(\mathrm{Gal})$ is the fixed Galactic absorption column density and $N_{\mathrm{H}}$ (Int) is the excess absorption. Spectral analysis was performed using XSPEC and fitting an absorbed power law where $\Gamma$ is the photon index. The X-ray decay slopes were calculated using non-linear least squares fitting with various broken power law models. For each case a simple non-broken power law provided the best fit. All errors given at 90 per cent confidence level apart from the X-ray decay slopes - they are given at $1 \sigma$.

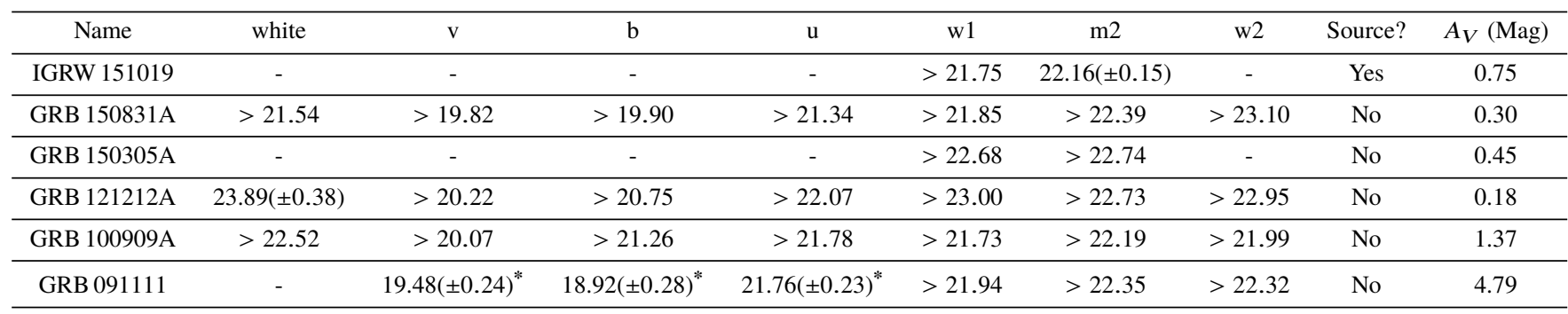

Table 4. UVOT multi-band magnitudes $(\mathrm{AB})$ and $3 \sigma$ upper limits of the 6 XRT detected sources. Filters ordered with decreasing wavelength.

* The GRB 091111 XRT position was within 10 arcsec of a very bright, saturated source and further analysis of the images suggest that the detections in the v, $\mathrm{b}$ and $\mathrm{u}$ filters are probably not real.

to the limited exposure from the ToOs but it is consistent with a decay slope of $\alpha \approx 1$ (see figure 1). An optimized fit could not be produced for the light curve so this decay slope is a rough estimation. Obtaining a spectrum is not possible due to the low number of counts detected: 102 in $6620 \mathrm{~s}$. This was a detection of a new GRB directly from Swift follow-up of a WEAK trigger and was not identified elsewhere (Starling 2015).

\subsection{IGRW 151019 - Active Galactic Nucleus candidate}

IGRW 151019 had showed no signs of fading after 4 weeks; initially the count rate was $4.0( \pm 2.7) \times 10^{-3} \mathrm{~s}^{-1}$ increasing by a factor of $\approx 1-8$ in the $\mathrm{X}$-ray band over a time period of $\approx 1$ month. There were only two observations of this source as a third ToO was not required as the source clearly was not fading. IGRW 151019 may therefore not be a GRB but a steady source. Analysis of the spectrum when fit with an absorbed power law gave a total column density,
$N_{\mathrm{H}}=4.0_{-3.0}^{+5.0} \times 10^{21} \mathrm{~cm}^{-2}$ and a photon index, $\Gamma=1.71 \pm 0.37$, which is broadly consistent with that of an AGN (Nandra \& Pounds 1994; Tozzi et al. 2006; Brightman \& Nandra 2011).

Inside the Swift XRT error circle lies the catalogued AllWISE source J193108.05+310756.4 (Cutri \& et al. 2014). The source is within 1.8 arcsec of the centre of the XRT position and within the 90 per cent XRT error region. The UVOT source we detected (table 4) for IGRW 151019 in the $\mathrm{m} 2$ filter has is RA, Dec (J2000) $292.78334,31.13252 \mathrm{deg}$ with a $1 \sigma$ positional error of 0.49 arcsec. A catalogued Gaia source has a position coincident to the UVOT source to within 0.5 arcsec. However, these sources lie just outside the 90 per cent XRT error region so we cannot confirm their association with the new X-ray transient. In addition, the Galactic extinction in this direction, $A_{V} \approx 0.75$. Examining the WISE source in more depth we find that its WISE colours, W1 $-\mathrm{W} 2=0.8$ and $\mathrm{W} 2-\mathrm{W} 3=2.4$, are consistent with that of an AGN (Mingo et al. 2016). It is possible that a transient event may have caused the initial 


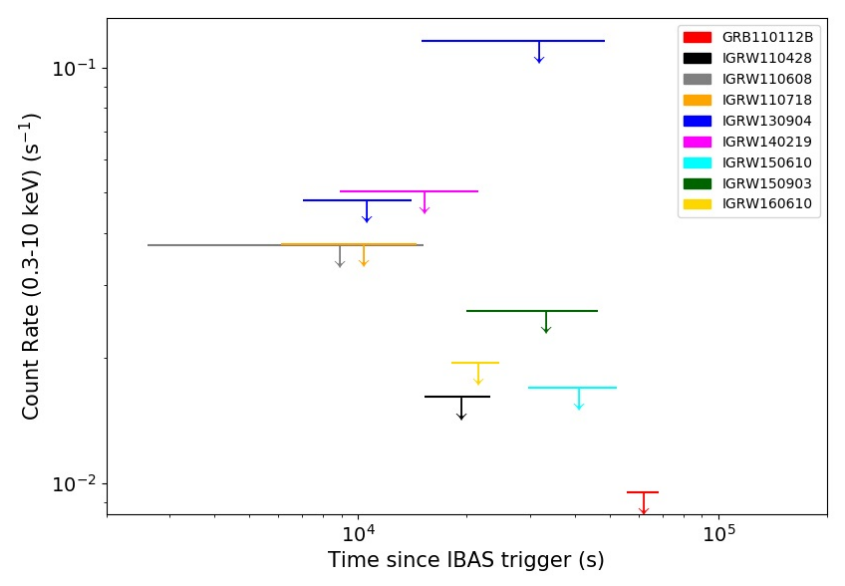

Figure 2. XRT $3 \sigma$ bayesian upper limits of the 9 non-detections out of our 15 selected WEAK triggers.

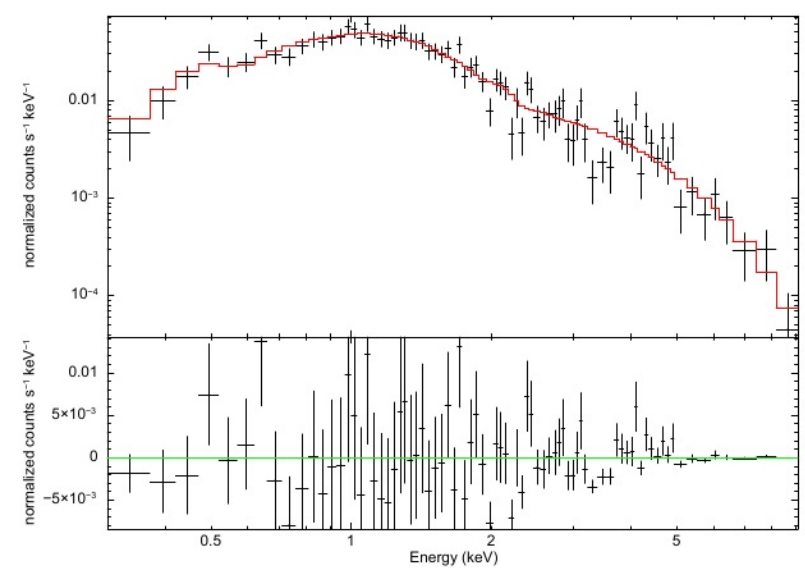

Figure 3. XRT spectrum of GRB 121212A with the best fitting absorbed power law model produced using XSPEC (red). The fit parameters can be seen in table 3 .

INTEGRAL WEAK trigger and the steady source may simply be a chance coincidence observation. However, this is unlikely and we conclude that IGRW 151019 is likely an AGN.

\section{IBAS AND SWIFT GRB SAMPLE PROPERTIES}

In section 1 we discussed that a low-luminosity GRB population could exist and that INTEGRAL may be capable of detecting it. Including the WEAK alert GRBs we have confirmed, the IBAS GRB sample size currently stands at 114 . The Swift sample size stands at 1060 GRBs with XRT detections for 846 GRBs (all values correct as of 2016 July 1 and Swift numbers were taken from the Swift GRB Table ${ }^{5}$ ).

Figure 6 shows the $\mathrm{T}_{90}$ and peak flux distributions of the INTEGRAL IBAS and Swift BAT samples. Analysis shows that the IBAS sample has a lower mean $\mathrm{T}_{90}$ ( $47 \mathrm{~s}$ compared to $70 \mathrm{~s}$ ). However, the Swift sample has a higher percentage of short GRBs

5 http : //swift.gsfc.nasa.gov/archive/grb_table/

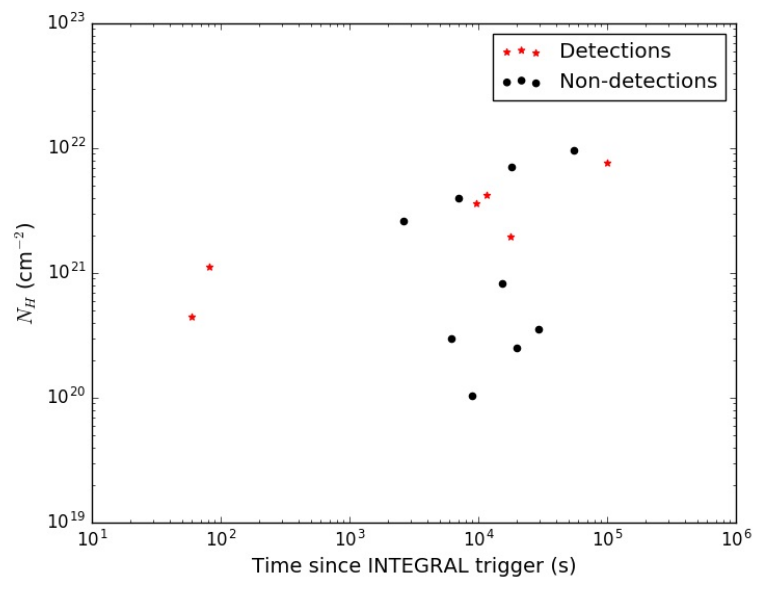

Figure 4. Plot of $T_{\mathrm{START}}$ against Galactic $N_{\mathrm{H}}$ of all of our 15 WEAK triggers including both XRT detections and non-detections.

compared to IBAS; 95 short GRBs out of the 992 Swift GRBs with measured $\mathrm{T}_{90}$ (9.6 per cent) compared to 6 short GRBs out of 114 INTEGRAL GRBs (5.3 per cent). The mean peak flux of the IBAS GRB sample is also lower than that of the Swift sample $\left(2.0 \mathrm{ph} \mathrm{cm}^{-2}\right.$ $\mathrm{s}^{-1}$ compared to $3.6 \mathrm{ph} \mathrm{cm}^{-2} \mathrm{~s}^{-1}$ ) meaning INTEGRAL routinely reaches lower peak flux values as a proportion of the total sample. With a lower average $T_{90}$ and peak flux it is more likely that the fluence distribution of INTEGRAL may be skewed towards fainter GRBs than Swift and with the addition of the lower IBIS sensitivity (discussed in section 1) INTEGRAL may be better suited to probing this lower luminosity GRB population.

\subsection{Can IBAS be used to probe low fluence GRBs?}

Our IBAS GRB sample contains 92 of the 114 IBAS GRBs, i.e. all those that have calculated fluence values. All IBAS $\mathrm{T}_{90}$ values were taken from the IBAS webpage ${ }^{1}$ but did not have any associated error limits. The IBAS GRB fluxes were calculated in XSPEC using a simple power law/cut-off power law model. The flux values were then multiplied by the $\mathrm{T}_{90}$ values to calculate the fluences. Several fluence values were taken from Vianello et al. (2009) and Bošnjak et al. (2014) and the properties of all IBAS GRBs with published and estimated measurements can be found in table A1.

For the IBAS GRBs detected by the XRT, we calculated the $\mathrm{X}$-ray flux values at 11 hours by fitting a series of single/broken power laws to the Swift X-ray afterglow light curves and performing an f-test to determine the best-fitting model. Once the best fitting model was obtained this was extrapolated to 39600 s (11 hours) and an estimate for the X-ray flux was determined. For the 1026 Swift GRBs with fluences, both the fluence, associated errors (at 90 per cent confidence) and X-ray flux at 11 hours values were taken from the NASA Goddard Space Flight Center Swift GRB Table ${ }^{1}$. X-ray flux values $<10^{-14} \mathrm{erg} \mathrm{cm}^{-2} \mathrm{~s}^{-1}$ were omitted as they were deemed too faint for Swift to detect and are therefore non-physical measurements. This resulted in 824 Swift GRBs and 33 IBAS GRBs with calculated X-ray flux values. It must be noted that the Swift GRB fluences are measured in the energy range of $15-150 \mathrm{keV}$ whereas the INTEGRAL GRB fluences are measured between $20-200 \mathrm{keV}$. We found that the flux, and therefore, fluence ratios between these two energy bands was $\frac{\mathrm{f}_{20-200}}{\mathrm{f}_{15-150}} \approx 1.22$ when 

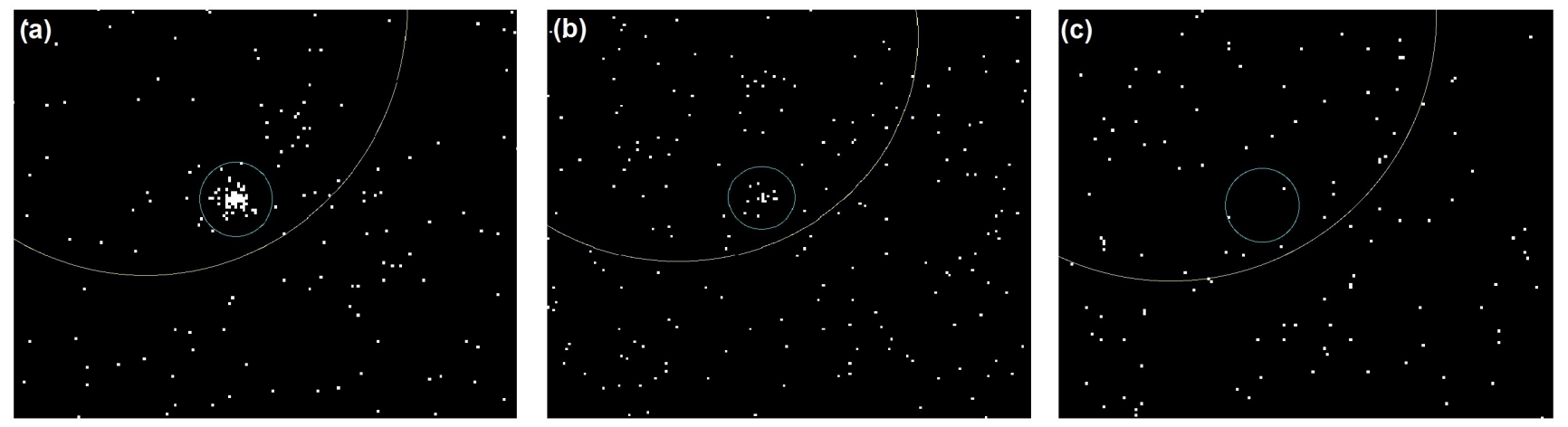

Figure 5. Images showing the X-ray source IGRW 150305/GRB 150305A (cyan) within the INTEGRAL error circle (yellow). The images correspond to observation times of $(1.7-2.7) \times 10^{4} \mathrm{~s}(\mathrm{a}),(1.2-1.3) \times 10^{5} \mathrm{~s}(\mathrm{~b})$ and $(7.0-7.4) \times 10^{5} \mathrm{~s}(\mathrm{c})$ after the GRB occurred with XRT exposure times of $3.0 \mathrm{ks}(\mathrm{a})$, $3.7 \mathrm{ks}$ (b) and $2.4 \mathrm{ks}$ (c) respectively. The point source clearly fades over time and is undetectable after $\approx 7.4 \times 10^{5} \mathrm{~s}$ ( $8-9$ days).
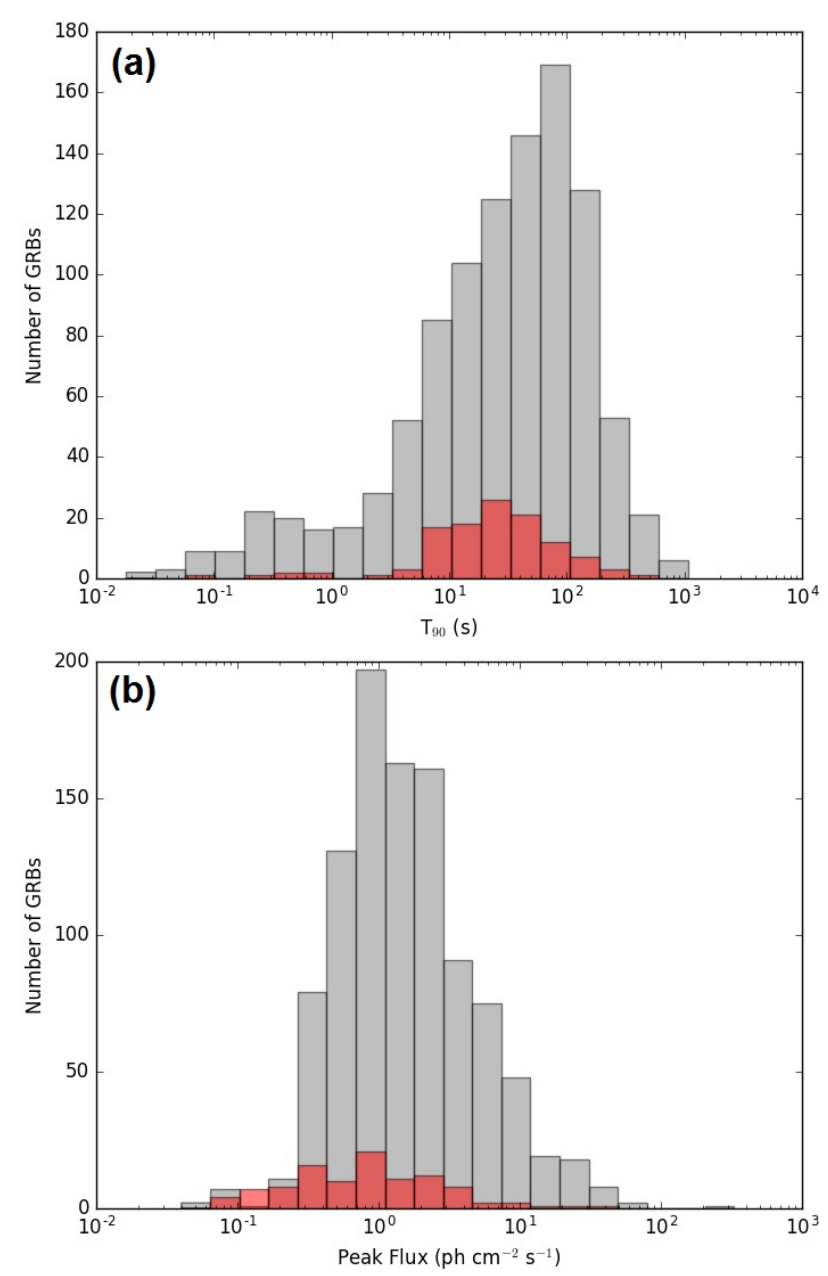

Figure 6. Histograms of $\mathrm{T}_{90}$ and peak flux distributions of both the Swift BAT (light grey) and IBAS (red) GRB samples. The peak flux values are measured between $15-150 \mathrm{keV}$ for Swift and $20-200 \mathrm{keV}$ for INTEGRAL. The fraction of short GRBs in the Swift and IBAS samples are 9.6 per cent and 5.3 per cent respectively.

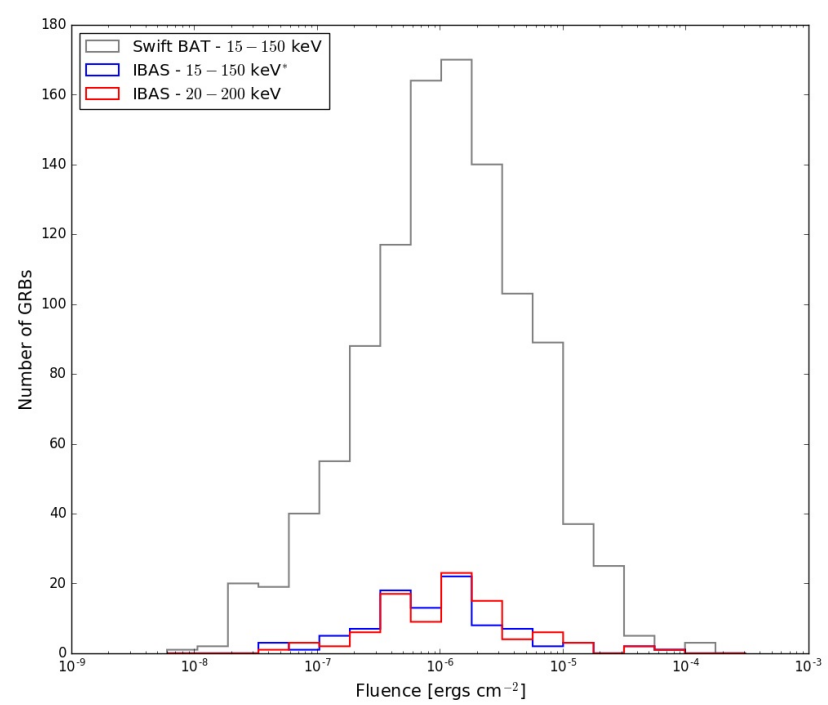

Figure 7. Fluence distributions of both the Swift BAT and the IBAS GRB samples. *Calculated using method described in section 4.1 .

measured from spectral fits for a small number of typical sources from our sample. We highlight that this is a mean ratio used to give an indication of the IBAS fluence values in the BAT energy band and will vary between GRBs within the sample. We also note that the $T_{90}$ can vary between different energy bands but we assumed that it remains constant for this conversion. Figure 7 shows the Swift BAT GRB fluence distribution overlaid with the IBAS GRB fluence distribution both in the $20-200 \mathrm{keV}$ and converted $15-150 \mathrm{keV}$ energy bands.

To determine if the IBAS and Swift GRB sample fluence distributions came from the same underlying population, a K-S test was performed on the Swift and IBAS fluence values in their respective $15-150$ and $20-200 \mathrm{keV}$ energy bands. If the test rejected the null hypothesis; that the underlying distribution of the samples was the same at a 95 per cent confidence level $(p<0.05)$ then it was assumed that the samples were not part of the same underlying distribution. The K-S test returned a $p$-value of 0.37 for the fluence distribution so we cannot reject the null hypothesis and we conclude that the INTEGRAL IBAS and Swift GRB samples most likely belong to the 
same distribution. Moreover, the mean fluence values are similar, $3.66 \times 10^{-6} \mathrm{erg} \mathrm{cm}^{-2}$ and $3.94 \times 10^{-6} \mathrm{erg} \mathrm{cm}^{-2}$ for the Swift and IBAS samples respectively. Converting the IBAS fluence values into the $15-150 \mathrm{keV}$ band using the ratio calculated previously gives a mean fluence value of $3.23 \times 10^{-6} \mathrm{erg} \mathrm{cm}^{-2}$ and when compared to the Swift distribution gives a K-S $p$-value of 0.06 . This results in the same conclusion as before; the two distributions most probably belong to the same fluence distribution.

We also analysed the correlations between GRB fluence, $\mathrm{T}_{90}$ and X-ray flux at 11 hours for both the IBAS and Swift GRB samples. The Spearman rank coefficients for fluence - $\mathrm{T}_{90}$ were $0.52( \pm 0.07)$ and $0.66( \pm 0.02)$ corresponding to $p$-values of $5.7 \times 10^{-8}$ and $3.8 \times 10^{-130}$ for the IBAS and Swift samples respectively. The Spearman rank coefficients for fluence - X-ray flux were $0.65( \pm 0.11)$ and $0.61( \pm 0.02)$ corresponding to $p$-values of $2.4 \times 10^{-5}$ and $3.96 \times 10^{-90}$ for the IBAS and Swift samples respectively. These values show these parameters exhibit significant correlation. Similar correlations have been reported in previous investigations (Gehrels et al. 2008; Evans et al. 2009; Margutti et al. 2012; Grupe et al. 2013). These authors acknowledge a wide spread in the data (to within an order of magnitude) due to a range of factors. We do not have available errors for the IBAS $\mathrm{T}_{90}$ values and these can be underestimated for very long GRBs. Our extrapolation values of X-ray flux at 11 hours do not have any associated errors and for some GRBs can only be taken as rough estimates due to the low number of data bins. Additionally we have used the observed X-ray flux, prior to correction for line-of-sight absorption. Although we have not fully accounted for these effects our correlations are significant. We conclude that our correlations agree with similar correlations from previous investigations.

Figure 8 shows the X-ray afterglows of the IBAS and Swift GRB samples. The plot highlights that the X-ray afterglow distribution of the IBAS sample sits comfortably within the Swift X-ray GRB afterglow distribution. The mean X-ray flux values at 11 hours for the IBAS and Swift samples are $2.85 \times 10^{-12} \mathrm{erg} \mathrm{cm}^{-2} \mathrm{~s}^{-1}$ and $1.48 \times 10^{-12} \mathrm{erg} \mathrm{cm}^{-2} \mathrm{~s}^{-1}$ showing that on average, the X-ray flux of the Swift sample GRBs is lower. However, only 54 IBAS GRBs were detected by the XRT; not all were followed up, some were non-detections, and only 33 were sufficiently sampled to obtain a value of X-ray flux at 11 hours.

Swift and INTEGRAL regularly detect similar fluence GRBs, however, the Swift sample has a low fluence, short GRB tail that the IBAS sample does not. Swift has also detected six GRBs which may belong to a further subclass of "ultra-long" GRBs where $\mathrm{T}_{90}$ values $\gtrsim 1000$ s (Gendre et al. 2013; Virgili et al. 2013; Evans et al. 2014; Levan et al. 2014; Cucchiara et al. 2015). However, with this low number of "ultra-long" GRBs $(<1 \%$ of the sample) we do not expect to have detected any with IBAS. Swift has detected $\approx 10$ times the number of GRBs than IBAS has detected. With such a large $S$ wift sample you would expect to see some very faint and very long GRBs and the differences in the distributions may arise from the smaller IBAS sample size and low number statistics. From this investigation we conclude that the Swift and IBAS GRB distributions are similar but not identical.

\section{CONCLUSIONS}

We investigated 15 INTEGRAL WEAK triggers utilising Swift for follow-up observations. Among these WEAK triggers, we confirm seven astrophysical events - six GRBs and one candidate AGN.
IGRW 150305 found directly from one of our chosen ToOs was identified as a GRB through this ToO campaign alone.

Comparisons of the fluence distributions of the full IBAS and Swift GRB samples showed that the two are similar but not identical. We also confirm correlations between the gamma-ray and X-ray properties found in previous investigations for both samples. Both the IBAS GRB fluence and X-ray afterglow light curve distributions comfortably lie within the Swift distributions. We conclude that Swift and IBAS typically reach similar fluence limits, while Swift appears to be more sensitive to short, low fluence GRBs.

We only sample $\approx 4$ per cent of the total WEAK trigger population. Hence we do not make any statistical statements for the total sample. We have shown that INTEGRAL can detect real GRB events below the STRONG threshold, along with other high energy transients and variables such as AGN. This allows future work to uncover the nature of yet more WEAK triggers to determine whether INTEGRAL can detect fainter GRBs.

\section{ACKNOWLEDGEMENTS}

The research leading to these results has received funding from the European Union's Horizon 2020 Programme under AHEAD project (grant agreement n. 654215). A. B. Higgins is supported by an STFC studentship. R. L. C. Starling and K. Wiersema acknowledge support from STFC. This work made use of data supplied by the UK Swift Science Data Centre at the University of Leicester which is supported by the UK Space Agency. S. Mereghetti acknowledges the support of ASI/INAF agreement No. 2016-025-R.0. We also thank the referee for useful comments and feedback.

During the reviewing process for this paper, our colleague Neil Gehrels passed away. We would like to acknowledge not only his input into this work, but his immense contributions to high energy astrophysics as a whole.

\section{REFERENCES}

Barthelmy S. D., 2004, in Flanagan K. A., Siegmund O. H. W., eds, Society of Photo-Optical Instrumentation Engineers (SPIE) Conference Series Vol. 5165, X-Ray and Gamma-Ray Instrumentation for Astronomy XIII. pp 175-189, doi:10.1117/12.506779

Bird A. J., et al., 2016, ApJS, 223, 15

Bošnjak Ž., Götz D., Bouchet L., Schanne S., Cordier B., 2014, A\&A, 561, A25

Breeveld A. A., et al., 2010, MNRAS, 406, 1687

Brightman M., Nandra K., 2011, MNRAS, 413, 1206

Burrows D. N., et al., 2005, Space Sci. Rev., 120, 165

Chapman R., Tanvir N. R., Priddey R. S., Levan A. J., 2007, MNRAS, 382, L21

Cline T. L., et al., 1999, A\&AS, 138, 557

Connaughton V., 2011, GRB Coordinates Network, 11569

Costa E., et al., 1997, Nature, 387, 783

Courvoisier T. J.-L., et al., 2003, A\&A, 411, L53

Cucchiara A., et al., 2015, ApJ, 812, 122

Cutri R. M., et al. 2014, VizieR Online Data Catalog, 2328

Daigne F., Mochkovitch R., 2007, A\&A, 465, 1

Evans P. A., et al., 2007, A\&A, 469, 379

Evans P. A., et al., 2009, MNRAS, 397, 1177

Evans P. A., et al., 2014, MNRAS, 444, 250

Foley S., McGlynn S., Hanlon L., McBreen S., McBreen B., 2008, A\&A, 484, 143

Gehrels N., Mészáros P., 2012, Science, 337, 932

Gehrels N., et al., 2004, ApJ, 611, 1005

Gehrels N., et al., 2008, ApJ, 689, 1161 


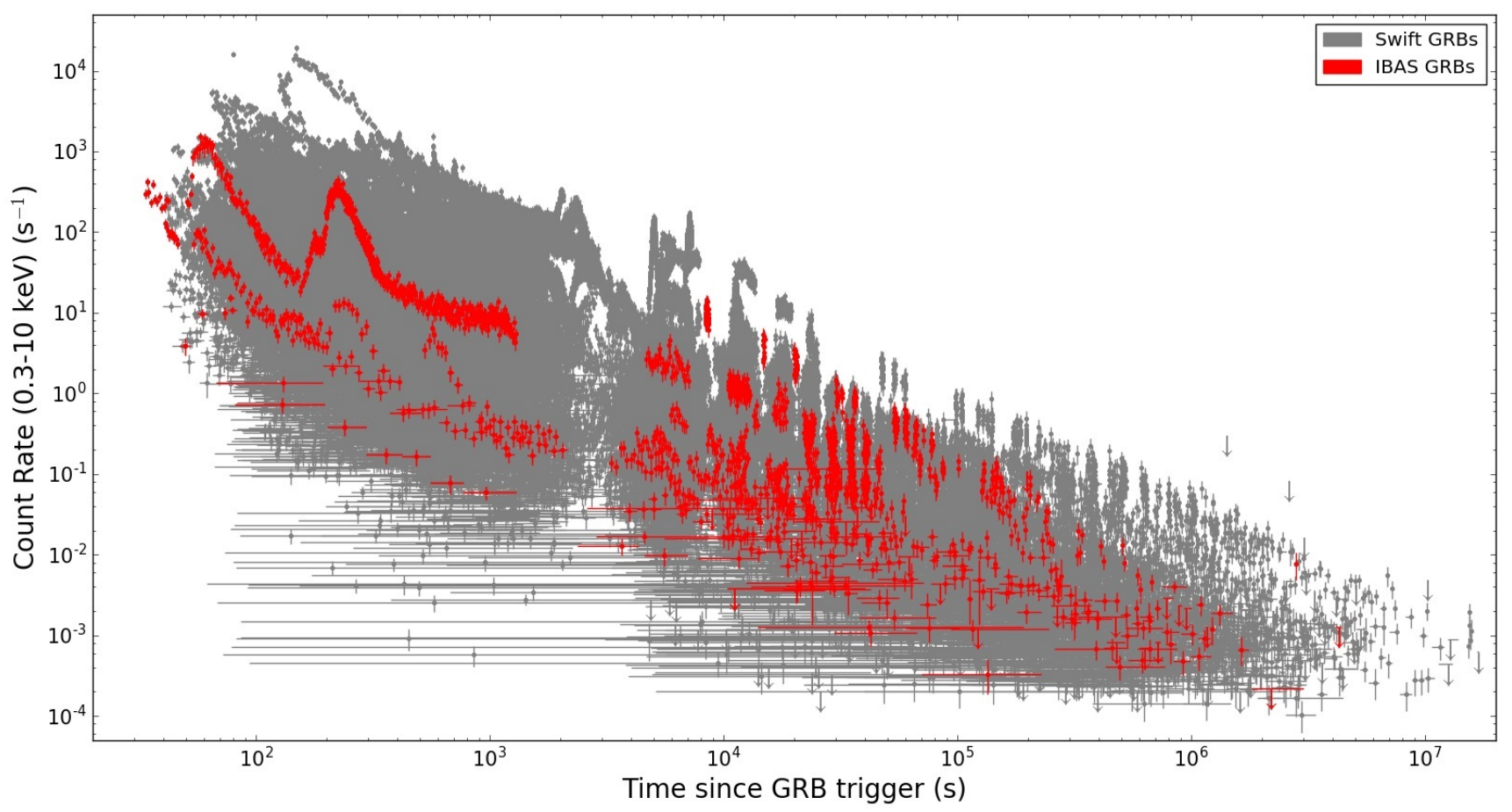

Figure 8. X-ray afterglows of the Swift GRB sample and the 54 IBAS GRBs observed by Swift/XRT.

Gendre B., et al., 2013, ApJ, 766, 30

Götz D., 2013, preprint, (arXiv: 1302 .4847)

Gotz D., Mereghetti S., Bozzo E., Ferrigno C., Pavan L., Borkowski J., 2016, GRB Coordinates Network, 19621

Grupe D., Nousek J. A., Veres P., Zhang B.-B., Gehrels N., 2013, ApJS, 209, 20

Kouveliotou C., Meegan C. A., Fishman G. J., Bhat N. P., Briggs M. S., Koshut T. M., Paciesas W. S., Pendleton G. N., 1993, ApJ, 413, L101

Kraft R. P., Burrows D. N., Nousek J. A., 1991, ApJ, 374, 344

Lebrun F., et al., 2003, A\&A, 411, L141

Levan A. J., et al., 2014, ApJ, 781, 13

Liang E., Zhang B., Virgili F., Dai Z. G., 2007, ApJ, 662, 1111

Margutti R., Zaninoni E., Bernardini M. G., Chincarini G., for the SwiftXRT team 2012, preprint, (arXiv: 1207.0537)

Mereghetti S., Götz D., Borkowski J., Walter R., Pedersen H., 2003, A\&A, 411, L291

Mereghetti S., Gotz D., Ferrigno C., Bozzo E., Ducci L., Borkowski J., 2015a, GRB Coordinates Network, 18210

Mereghetti S., Gotz D., Ferrigno C., Bozzo E., Saral G., Borkowski J., 2015b, GRB Coordinates Network, 18623

Mereghetti S., Gotz D., Ferrigno C., Bozzo E., Favre T., Borkowski J., 2016, GRB Coordinates Network, 19046

Mészáros P., Rees M. J., 1997, ApJ, 476, 232

Mingo B., et al., 2016, MNRAS, 462, 2631

Nandra K., Pounds K. A., 1994, MNRAS, 268, 405

Norris J. P., 2002, ApJ, 579, 386

Norris J. P., Bonnell J. T., Kazanas D., Scargle J. D., Hakkila J., Giblin T. W., 2005, ApJ, 627, 324

O'Brien P. T., et al., 2006, The Astrophysical Journal, 647, 1213

Pescalli A., et al., 2016, A\&A, 587, A40

Piran T., 2003, Nature, 422, 268

Roming P. W. A., et al., 2005, Space Sci. Rev., 120, 95

Sazonov S. Y., Lutovinov A. A., Sunyaev R. A., 2004, Nature, 430, 646

Schlafly E. F., Finkbeiner D. P., 2011, ApJ, 737, 103

Soderberg A. M., et al., 2004, Nature, 430, 648

Starling R., 2015, GRB Coordinates Network, 17551
Tozzi P., et al., 2006, A\&A, 451, 457

Ubertini P., et al., 2003, A\&A, 411, L131

Vedrenne G., et al., 2003, A\&A, 411, L63

Vianello G., Götz D., Mereghetti S., 2009, A\&A, 495, 1005

Virgili F. J., et al., 2013, ApJ, 778, 54

Wijers R. A. M. J., Rees M. J., Meszaros P., 1997, MNRAS, 288, L51

Willingale R., Starling R. L. C., Beardmore A. P., Tanvir N. R., O'Brien P. T., 2013, MNRAS, 431, 394

Winkler C., et al., 2003, A\&A, 411, L1

Woosley S. E., Heger A., 2006, ApJ, 637, 914

von Kienlin A., et al., 2003, A\&A, 411, L299

\section{APPENDIX A: SUPPLEMENTARY TABLES}




\begin{tabular}{|c|c|c|c|}
\hline Name & $\begin{array}{c}\text { Fluence }[20-200 \mathrm{keV}]\left(10^{-7}\right. \\
\left.\mathrm{erg} \mathrm{cm}^{-2}\right)\end{array}$ & $\begin{array}{c}\text { X-ray Flux at } 11 \text { hours } \\
{[0.3-10 \mathrm{keV}]\left(10^{-12} \mathrm{erg}\right.} \\
\left.\mathrm{cm}^{-2} \mathrm{~s}^{-1}\right)\end{array}$ & $\mathrm{T}_{90}(\mathrm{~s})$ \\
\hline GRB $030227^{*}$ & $6.10_{-5.90}^{+3.50}$ & - & 15 \\
\hline GRB $030320^{*}$ & $54.2_{-11.7}^{+13.3}$ & - & 48 \\
\hline GRB $030501^{*}$ & $17.2_{-3.10}^{+1.60}$ & - & 25 \\
\hline GRB $030529^{\#}$ & 0.52 & - & 16 \\
\hline GRB $031203^{*}$ & $10.6_{-3.00}^{+2.70}$ & - & 19 \\
\hline GRB $040106^{*}$ & $95.0_{-30.0}^{+23.0}$ & - & 47 \\
\hline GRB $040223^{*}$ & $27.2_{-1.90}^{+0.80}$ & - & 258 \\
\hline GRB $040323^{*}$ & $20.6_{-2.90}^{+2.30}$ & - & 14 \\
\hline GRB $040403^{*}$ & $4.00_{-3.70}^{+1.60}$ & - & 15 \\
\hline GRB $040422^{*}$ & $4.90_{-3.60}^{+1.00}$ & - & 10 \\
\hline GRB $040624^{\#}$ & 4.81 & - & 27 \\
\hline GRB $040730^{*}$ & $6.30_{-3.30}^{+4.40}$ & - & 42 \\
\hline GRB $040812^{\#}$ & 1.40 & - & 8 \\
\hline GRB $040827^{*}$ & $11.1_{-4.00}^{+2.80}$ & - & 32 \\
\hline GRB $040903^{\#}$ & 0.96 & - & 7 \\
\hline GRB 041015 \# & 5.12 & - & 30 \\
\hline GRB $041218^{*}$ & $58.2_{-3.70}^{+3.50}$ & - & 38 \\
\hline GRB 041219A* & $867_{-129}^{+0.50}$ & - & 239 \\
\hline GRB 050129\# & 4.10 & - & 30 \\
\hline GRB 050223 & $10.8_{-2.10}^{+2.70}$ & 0.19 & 30 \\
\hline GRB 050502A* & $13.9_{-4.00}^{+1.10}$ & - & $>11$ \\
\hline GRB 050504* & $10.0_{-4.50}^{+4.10}$ & - & 44 \\
\hline GRB $050520^{*}$ & $16.6_{-5.00}^{+4.90}$ & 0.20 & 52 \\
\hline GRB 050522\# & 0.69 & - & 11 \\
\hline GRB 050525A* & $154_{-8.40}^{+5.70}$ & 1.5 & 9 \\
\hline GRB $050626^{*}$ & $6.30_{-1.00}^{+0.40}$ & - & 52 \\
\hline GRB 050714A & $5.58_{-1.84}^{+2.75}$ & - & 34 \\
\hline GRB $050918^{*}$ & $30.2_{-9.0}^{+10.5}$ & - & 280 \\
\hline GRB 050922A & 0.59 & - & 10 \\
\hline GRB 051105B* & $2.80_{-2.00}^{+1.50}$ & - & 14 \\
\hline GRB 051211B* & $16.1_{-3.30}^{+4.60}$ & 0.92 & 47 \\
\hline GRB 060114* & $16.0_{-3.30}^{+4.60}$ & - & 80 \\
\hline GRB $060130^{\#}$ & 2.25 & - & 19 \\
\hline GRB 060204A* & $4.80_{-3.30}^{+2.40}$ & - & 52 \\
\hline GRB 060428C* & $18.6_{-3.90}^{+2.20}$ & - & 10 \\
\hline GRB 060901* & $62.2_{-5.90}^{+3.50}$ & 1.2 & 16 \\
\hline GRB $060930^{\#}$ & 2.63 & - & 9 \\
\hline GRB 060912B* & $12.0_{-5.10}^{+5.80}$ & - & 140 \\
\hline GRB $061025^{*}$ & $10.1_{-4.80}^{+1.30}$ & 0.14 & 11 \\
\hline
\end{tabular}




\begin{tabular}{|c|c|c|c|}
\hline Name & $\begin{array}{c}\text { Fluence }[20-200 \mathrm{keV}]\left(10^{-7}\right. \\
\left.\mathrm{erg} \mathrm{cm}^{-2}\right)\end{array}$ & $\begin{array}{c}\text { X-ray Flux at } 11 \text { hours } \\
{[0.3-10 \mathrm{keV}]\left(10^{-12} \mathrm{erg}\right.} \\
\left.\mathrm{cm}^{-2} \mathrm{~s}^{-1}\right)\end{array}$ & $\mathrm{T}_{90}(\mathrm{~s})$ \\
\hline GRB $061122^{*}$ & $155_{-5.30}^{+3.40}$ & 2.2 & 12 \\
\hline GRB 070309 & $4.93_{-1.98}^{+3.12}$ & - & 22 \\
\hline GRB $070311^{*}$ & $23.6_{-5.30}^{+1.70}$ & 1.22 & 32 \\
\hline GRB 070615 & 2.01 & - & 15 \\
\hline GRB 070707 & $3.58_{-1.94}^{+4.04}$ & - & 0.7 \\
\hline GRB $070925^{*}$ & $36.1_{-3.40}^{+1.70}$ & - & 19 \\
\hline GRB 071003 & $94.6_{-2.96}^{+4.22}$ & 3.5 & 38 \\
\hline GRB 071109* & $3.60_{-3.50}^{+4.00}$ & - & 30 \\
\hline GRB 080120 & $13.2_{-7.67}^{+17.0}$ & 0.13 & 15 \\
\hline GRB 080603A & $\begin{array}{r}12.3_{-5.90}^{+1.70} \\
\end{array}$ & 1.5 & 150 \\
\hline GRB 080613A* & $12.3_{-5.90}^{+1.70}$ & - & 30 \\
\hline GRB 080723B* & $396_{-6.70}^{+6.70}$ & 12.6 & 95 \\
\hline GRB 080922* & $17.3_{-6.50}^{+6.90}$ & - & 60 \\
\hline GRB 081003B* & $26.2_{-24.5}^{+2.00}$ & - & 20 \\
\hline GRB $081016^{*}$ & $22.0_{-4.50}^{+1.40}$ & - & 30 \\
\hline GRB 081204* & $5.10_{-4.80}^{+5.10}$ & - & 12 \\
\hline GRB 090107B* & $12.4_{-4.60}^{+1.30}$ & 0.73 & 15 \\
\hline GRB 090625B* & $12.4_{-2.00}^{+1.20}$ & 0.38 & 8 \\
\hline GRB 090702 & $1.93_{-0.81}^{+1.44}$ & - & 6 \\
\hline GRB 090704* & $54.0_{-8.00}^{+4.90}$ & - & 70 \\
\hline GRB 090814B* & $15.1_{-2.40}^{+2.30}$ & 1.4 & 42 \\
\hline GRB 090817* & $\begin{array}{r}18.7_{-9.80}^{+10.9} \\
\end{array}$ & 2.4 & 30 \\
\hline GRB 091111 & $20.0_{-0.82}^{+5.90}$ & - & 100 \\
\hline GRB 091202 & $7.03_{-2.35}^{+3.02}$ & - & 25 \\
\hline GRB 091230 & $17.9_{-9.57}^{+20.5}$ & - & 70 \\
\hline GRB $100103 A^{*}$ & $52.5_{-4.00}^{+2.10}$ & 2.1 & 30 \\
\hline GRB $100518 A^{*}$ & $5.20_{-3.80}^{+4.40}$ & 0.87 & 25 \\
\hline GRB 100713A & $5.65_{-1.80}^{+2.65}$ & 0.20 & 20 \\
\hline GRB 100909A & $21.5_{-4.70}^{+7.00}$ & 0.26 & 60 \\
\hline GRB 101112A* & $21.1_{-7.40}^{+4.40}$ & 0.50 & 6 \\
\hline GRB 110206A & $17.2_{-6.10}^{+11.6}$ & 2.0 & 15 \\
\hline GRB $110708 A^{*}$ & $24.8_{-4.60}^{+1.90}$ & - & 50 \\
\hline GRB 110903A* & $148_{-17.5}^{+11.9}$ & 3.8 & 430 \\
\hline GRB 120202A & $8.00_{-7.70}^{+2.10}$ & - & 70 \\
\hline GRB 120419A & $3.88_{-2.49}^{+6.18}$ & - & 15 \\
\hline GRB 120711A & $\begin{array}{r}440_{-5.00}^{+50.0} \\
\end{array}$ & 40 & 135 \\
\hline GRB 121102A & $24.1_{-8.10}^{+12.4}$ & 0.56 & 25 \\
\hline GRB 121212A & 1.50 & 0.46 & 10 \\
\hline
\end{tabular}




\section{A.B. Higgins et al.}

\begin{tabular}{cccc}
\hline Name & $\begin{array}{c}\text { Fluence }[20-200 \mathrm{keV}]\left(10^{-7}\right. \\
\left.\mathrm{erg} \mathrm{cm}{ }^{-2}\right)\end{array}$ & $\begin{array}{c}\text { X-ray Flux at 11 hours } \\
{[0.3-10 \mathrm{keV}]\left(10^{-12} \mathrm{erg}\right.} \\
\left.\mathrm{cm}^{-2} \mathrm{~s}^{-1}\right)\end{array}$ & $T_{90}(\mathrm{~s})$ \\
\hline GRB 130513A & $17.0_{-6.50}^{+10.3}$ & - & 50 \\
\hline GRB 130514B & $10.2_{-6.20}^{+14.4}$ & 1.6 & 10 \\
\hline GRB 130903A & $17.1_{-5.40}^{+8.10}$ & - & 70 \\
\hline GRB 131122A & $24.8_{-8.20}^{+12.3}$ & - & 80 \\
\hline GRB 140206A & $16.0_{-3.00}^{+3.00}$ & 9.2 & $>60$ \\
\hline GRB 140320B & $12.7_{-5.94}^{+11.8}$ & 0.55 & 100 \\
\hline GRB 140320C & 3.52 & - & 30 \\
\hline GRB 140815A & $5.00_{-2.59}^{+5.10}$ & - & 4 \\
\hline GRB 141004A & $6.92_{-3.40}^{+6.88}$ & 0.09 & 60 \\
\hline GRB 150219A & $57.1_{-11.2}^{+14.9}$ & 0.62 & 100 \\
\hline GRB 150305A & $12.1_{-6.45}^{+14.2}$ & - & 2 \\
\hline GRB 150831A & $\approx 3$ & 0.33 & 50 \\
\hline GRB 151120A & $\approx 20$ & 0.66 & 10 \\
\hline GRB 160221A & $\approx 5$ & - & 100 \\
\hline GRB 160629A & $\approx 60$ & - & \\
\hline
\end{tabular}

Table A1. The prompt emission fluence, $\mathrm{T}_{90}$ and X-ray afterglow flux of all 92 IBAS GRBs within our sample. All $\mathrm{T}_{90}$ values were taken from IBAS ${ }^{1}$. Some GRBs were not observed by Swift or had very poorly sampled Swift XRT afterglows and therefore an X-ray flux at 11 hours could not be obtained. Some fluence values contain no errors as the fitted model would not converge and would not provide an error on the normalisation. This meant no error could be found on the flux values and therefore the fluence values but the values should still be representative of the actual fluence. Fluence values for GRB 150831, GRB 151120A, GRB 160221A, GRB 160629A are approximations from the GCN Circulars (Mereghetti et al. 2015a,b, 2016; Gotz et al. 2016) as the spectral data were not yet public.

${ }^{*}$ Fluence values taken from Bošnjak et al. (2014).

\# Fluence values taken from Vianello et al. (2009). 